\title{
Primer registro de Eratyrus mucronatus Stal, 1859: vector de la enfermedad de Chagas en el departamento del Putumayo, Colombia
}

\section{First report of Eratyrus mucronatus Stal, 1859: vector of Chagas disease in the department of Putumayo, Colombia Primeiro registro de Eratyrus mucronatus Stal, 1859: vetor da
doença de Chagas no departamento de Putumayo, Colômbia}

Keila Ortiz - Canamejoy, Biol., Esp.*

\section{Sra. Editora,}

La enfermedad de Chagas, también llamada tripanosomiasis americana, es una enfermedad potencialmente mortal causada por el parásito protozoo Trypanosoma cruzi. Esta enfermedad es un problema de salud pública, ya que se calcula que en el mundo hay entre 6 y 7 millones de personas infectadas por este parásito; esta patología se encuentra sobre todo en zonas endémicas de 21 países de América Latina (1), incluido Colombia, donde la principal vía de transmisión es vectorial (2). De acuerdo con los datos del Sistema de Vigilancia Epidemiológica (SIVIGILA) para Putumayo, desde el año 2012 a la semana epidemiológica 45 del 2018, se encuentran notificados 21 casos de chagas crónicos y 8 casos de chagas agudos. A partir de esto se podría suponer que existen muchos casos de chagas que faltan por identificarse (3).

En América Latina, el parásito T. cruzi se transmite principalmente por contacto con las heces $\mathrm{u}$ orina infectadas de triatominos que se alimentan de sangre. En general, pican en una zona expuesta de la piel, como la cara, y defecan cerca de la picadura; los parásitos penetran en el organismo cuando la persona picada se frota instintivamente y empuja las heces o la orina hacia la picadura, los ojos, la boca o alguna lesión cutánea abierta (4). T. cruzi también se puede transmitir por consumo de alimentos contaminados; por la transfusión de sangre infectada; por la transmisión de la madre infectada a su hijo durante el embarazo o el parto; por el transplante de órganos provenientes de una persona infectada; y por accidentes de laboratorio (4).

Los triatominos son conocidos en el departamento del Putumayo como "Pitos". Estos juegan un papel vital en la transmisión de la triapanosomiasis Americana; afectan principalmente las áreas rurales en donde las condiciones ecológicas, aunadas a las condiciones

* Bióloga, Especialista en Epidemiología, Profesional de Apoyo del área de Entomología y Epidemiología del Laboratorio de Salud Pública, Secretaría de Salud del departamento del Putumayo. Mocoa, Putumayo, Colombia.

Correspondencia: Keila Ortiz Canamejoy, 860001. Mocoa, Putumayo, Colombia. E-mail: keilaortiz70@gmail.com 
tradicionales culturales de los habitantes y las precarias condiciones socioeconómicas, contribuyen a que el vector conviva en la vivienda con los humanos y mamíferos reservorios domésticos (perros, gatos); así se propicia la continuidad de esta endemia (5).

Por este motivo resulta de gran interés compartir con los lectores de la revista MedUNAB la experiencia del primer registro del vector de chagas Eratyrus mucronatus Stal, 1859 (Hemiptera: Reduviidae) presente en el departamento del Putumayo, identificado en la vigilancia entomológica realizada por el Laboratorio de Salud Pública de la Secretaría de Salud departamental de Putumayo.

El departamento del Putumayo se encuentra localizado al suroccidente del territorio colombiano. Cerca del 9\% del territorio departamental pertenece a la unidad de Paisaje Cordillera de los Andes, ubicada por encima de los 900 metros sobre el nivel del mar (m.s.n.m.m); por otra parte, cerca del $6 \%$ pertenece a la unidad de Paisaje Piedemonte, ubicada entre los 300 y los 900 m.s.n.m.m y cerca del $85 \%$ pertenece a la unidad Llanura Amazónica, ubicada por debajo de los 300 m.s.n.m.m. La mayor altitud se registra en el cerro Patascoy, con 3950 m.s.n.m.m, y la menor se encuentra en el extremo oriental del municipio de Puerto Leguízamo, con 150 m.s.n.m.m (6). El departamento del Putumayo cuenta con 13 municipios de los cuales 9 presentan las condiciones ambientales, geográficas y climáticas adecuadas para la sobrevivencia de insectos vectores. Estos municipios son Orito, Mocoa, Puerto asís, San Miguel, Valle del Guamuez, Puerto Guzmán, Villagarzón, Puerto Caicedo y Puerto Leguízamo (7).

El municipio de Orito se encuentra localizado a los $0^{\circ} 38^{\prime}$ de latitud Norte y $76^{\circ} 37^{\prime}$ de latitud Oeste de Greenwich, a una altura entre los 250 y 3,500 m.s.n.m.m; cuenta con un área de $1,862,36 \mathrm{Km}^{2}$ (IGAC, 2001), con una temperatura promedio de $25^{\circ} \mathrm{C}$ y una humedad relativa de $88 \%$. El municipio tiene una población de 49,420 habitantes, distribuida en la zona urbana con 23,278 personas y en la zona rural con 26,142. El municipio presenta 9 inspecciones de policía, conformadas por 124 veredas (incluye asentamientos afrodescendientes en proceso de constitución) y 34 comunidades indígenas distribuidas en 12 resguardos y 22 cabildos (8) (Figura 1).

La Secretaria de Salud del Departamento del Putumayo recibió el reporte del Sistema de Vigilancia Epidemiológica (SIVIGILA) de un caso de chagas

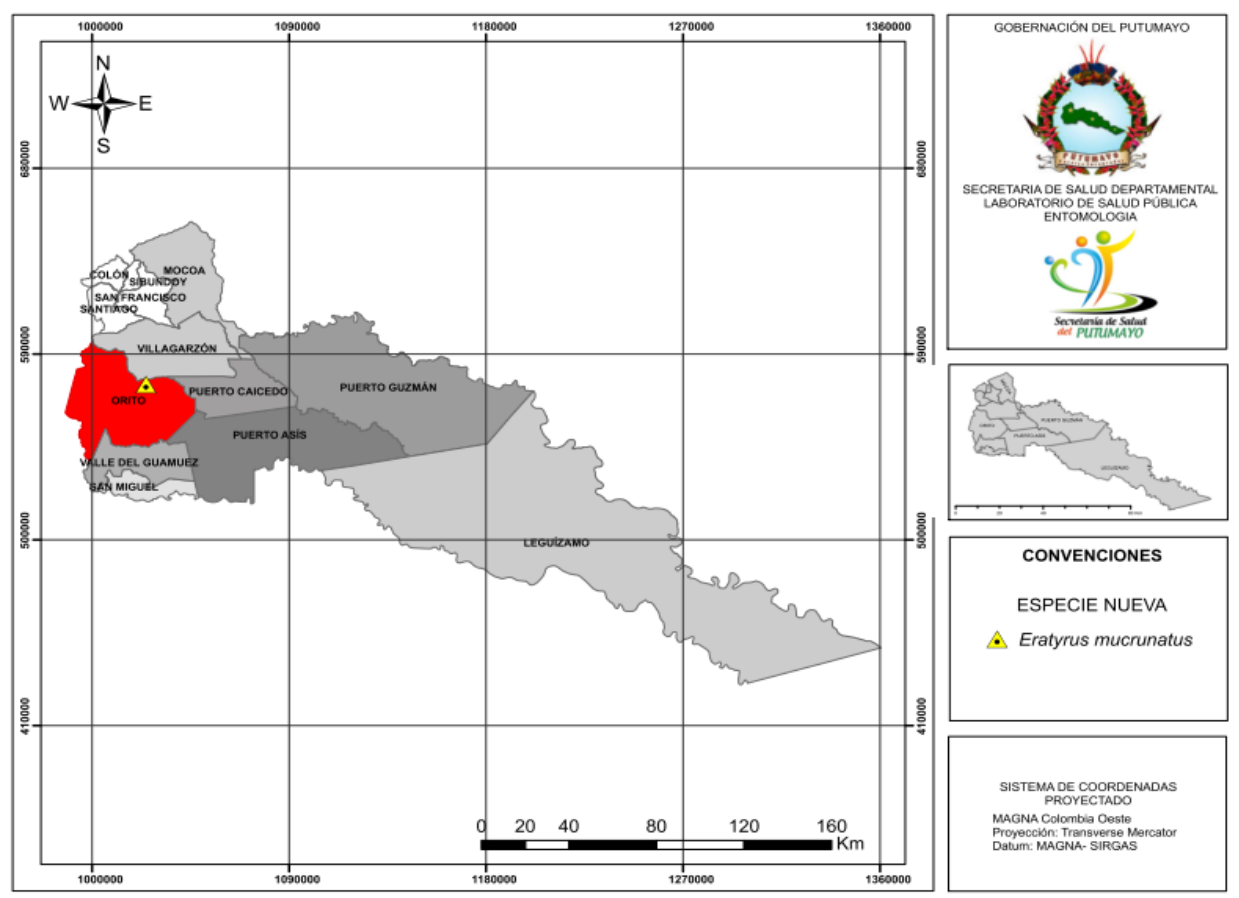

Figura 1. Mapa de la ubicación geográfica del municipio de Orito en el departamento del Putumayo

Fuente: Elaborado por el área de Sistemas de Información Geográfica de la Gobernación del Putumayo, con información suministrada por el área de entomología del Laboratorio de Salud Pública de Putumayo 2018. 
agudo en una paciente menor de edad, que pertenece al Cabildo indígena Awá la Turbia, del municipio de Orito (Figura 2). Tan pronto se obtuvo el reporte, el Laboratorio de entomología del Laboratorio de Salud Pública (LDSP) — con el apoyo del Programa de ETV - realizaron una búsqueda activa del vector casa a casa, capacitación a la población en el reconocimiento del vector, sitios de cría, técnica de recolección de los insectos, métodos de transmisión de la enfermedad, signos y síntomas de la enfermedad. Sin embargo, durante la búsqueda no se colectaron insectos vectores.

Posteriormente se instaló un Puesto de Recolección de Triatominos, ubicado en la vivienda del gobernador indígena del cabildo, con el fin de que las personas que identifiquen triatominos los colecten y los envíen al Laboratorio de entomología del LDSP para su identificación taxonómica. En el primer envío, el Laboratorio de Entomología recepcionó tres triatominos; la familia de la paciente que presentó Chagas agudo, colectó en el intradomicilio dos de ellos: uno identificado como Rhodnius prolixus y otro Eratyrus mucronatus; de la misma manera, el gobernador indígena de la comunidad envió otro triatomino colectado en el intradomicilio, identificado como Rhodnius prolixus. En el segundo envío el Laboratorio de Entomología recepcionó de una familia, 3 triatominos y de una segunda familia un triatomino. Las dos familias manifiestan haberlos capturado en el intradomicilio; los 4 triatominos fueron identificados como Rhodnius prolixus, especies vectoras de la enfermedad de chagas. La identificación taxonómica de estas especies fue realizada por la profesional del laboratorio de Entomología del LDSP, mediante la clave de Lent \& Wygodzinsky 1979; Los especímenes se encuentran en el registro de muestras entomológicas del año 2017 con los códigos 47, 73, 198 y 199. Como control de calidad indirecto, se envió al Grupo de Entomología del Instituto Nacional de Salud una muestra de cada especie identificadas como: Eratyrus mucronatus y Rhodnius prolixus, para la confirmación de las especies, con un resultado del $100 \%$ de concordancia. A los insectos no se les realizaron pruebas parasitológicas de infección de Trypanosoma cruzi debido a que llegaron muertos y no estaban alimentados.

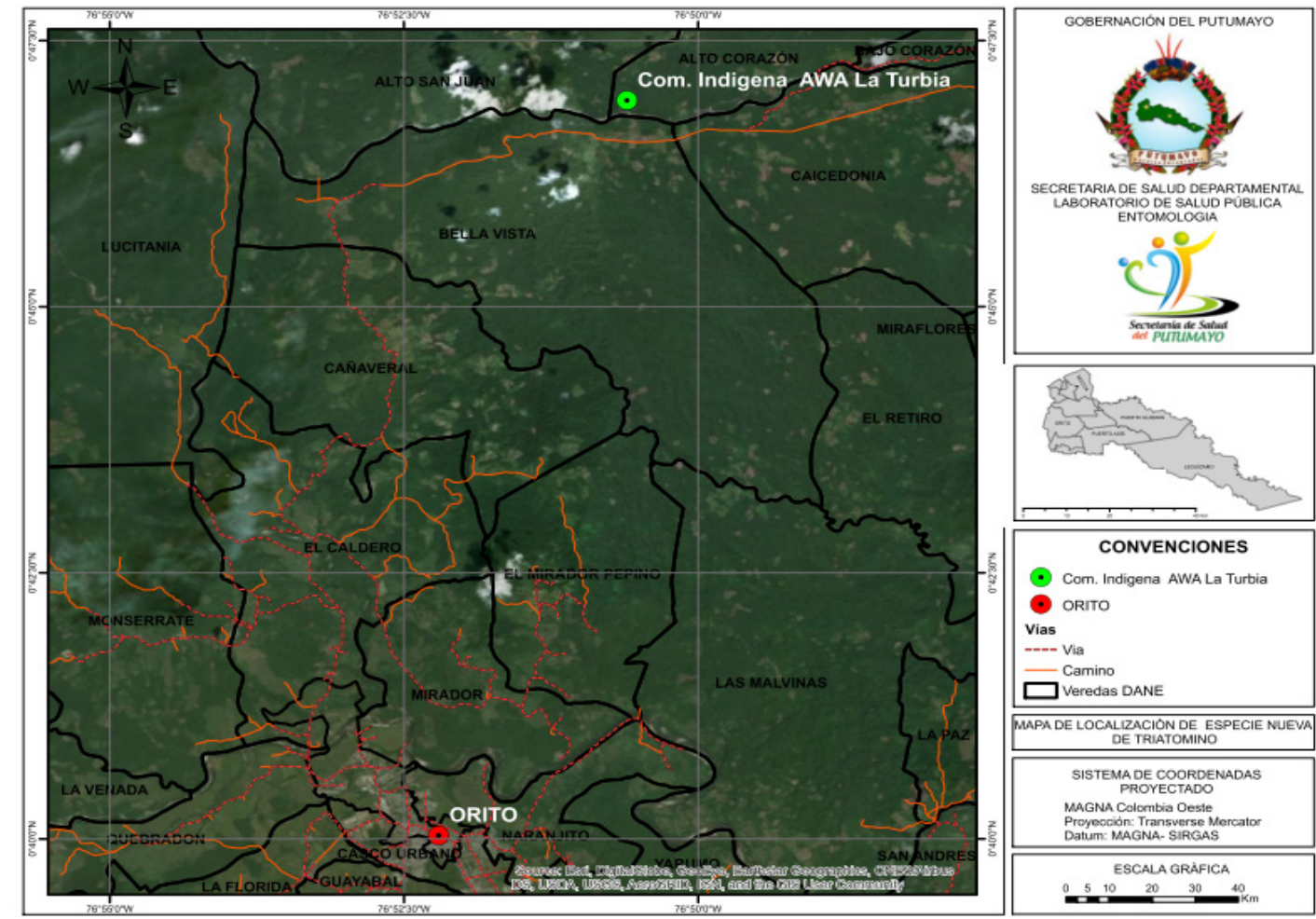

Figura 2. Ubicación Geográfica del Resguardo indígena Awá la Turbia del municipio de Orito

Fuente: Elaborado por el área de Sistemas de Información Geográfica de la Gobernación del Putumayo, con información suministrada por el área de entomología del Laboratorio de Salud Pública de Putumayo 2018. 
Una vez identificados los vectores en la zona, la Secretaría de Salud del departamento del Putumayo, realizó acciones de control correspondientes, para prevenir otros casos de Chagas.

Aunque varios artrópodos han sido experimentalmente infectados con Trypanosoma cruzi (Chagas, 1909), solo los triatominos son epidemiológicamente importantes en la transmisión de la enfermedad de Chagas. Los triatominos forman una subfamilia de los depredadores redúvidos, caracterizados por su hábito hematófago obligado. Actualmente existen 123 especies de triatominos, agrupados en 5 tribus y 14 géneros $(9,10)$. En Colombia existen 24 especies de triatominos, 15 se han encontrado con infecciones naturales por T. cruzi: Eratyrus mucronatus, Eratyrus cuspidatus Panstrongylus geniculatus, Panstrongylus lignarius, Panstrongylus rufotuberculatus, $T$. dimidiata, Triatoma dispar, T. maculata, T. venosa, $R$. brethesi, $R$. colombiensis, $R$. pallescens, $R$. pictipes, R. prolixus, Cavernicola pilosa (10).

En el departamento del Putumayo, según estudios publicados por Guhl et al. (11), Barreto et al. (12) y Vásquez et al. (13), hasta el momento se habían reportado tres especies vectoras de triatominos como: Panstrongylus geniculatus, Rhodnius pictipes, y Rhodnius prolixus. Se destaca en esta investigación la presencia del nuevo registro de Eratyrus mucronatus (macho), el cual fue colectado en el cabildo indígena Awá La Turbia, del municipio de Orito, el 12 de Junio de 2017. Esta especie ha sido reportado en ocho departamentos de Colombia como Antioquia, Arauca, Boyacá, Casanare, Cundinamarca, Meta, Norte de Santander y Vaupés (14), ampliándose así su distribución en el país. Este hecho resalta su importancia en Salud Pública por ser vector de la enfermedad de chagas. La introducción de estas especies vectoras en las viviendas se debe a que el hombre penetró en los focos naturales; su acción sobre los ecotopos silvestres fue y sigue siendo devastadora por la quema y tala de los bosques, la construcción de viviendas con material vegetal y la caza indiscriminada de animales silvestres. Como consecuencia, se ha modificado en muchas zonas del país la relación endémica natural de los componentes biológicos que intervienen en el ciclo del parásito, así como el comportamiento de los insectos triatominos, los cuales son primitivamente silvestres (15). De acuerdo a los diferentes estudios realizados por Sherlock et al. (16), se confirma que E. mucronatus ha sido encontrada naturalmente infectado por T. cruzi y normalmente en hábitats como nidos de mamíferos y palmas. Además, ha sido relacionado con Didelphis marsupialis (17) como fuente de alimentación en triatominos; datos relevantes, ya que, según Perruolo y Morales (18), este vertebrado es considerado el principal reservorio de la enfermedad de chagas en la zona norte del estado de Táchira. En Bolivia, fue encontrado en un domicilio e infectado por T. cruzi $(19,20)$; en Colombia, Molina et al. (21) reportan que se encuentra en ambiente domiciliado.

De acuerdo con Caravallo et al. (20), que clasifican a E. mucronatus como ocasionalmente domiciliario, esta especie puede adaptarse con relativa rapidez a ecotopos artificiales estables, mientras sus hábitats naturales sean destruidos por acciones del hombre o por otras acciones como: incremento poblacional, enemigos naturales o insuficiencia de fuentes alimentarias (22).

La otra especie encontrada en este estudio es Rhodnius prolixus, considerada una de las especies de mayor importancia para Colombia desde el punto de vista epidemiológico, debido a la frecuencia con que coloniza el domicilio humano y el alto porcentaje de infección por T.cruzi (11).

La actualización de la distribución de triatominos en Colombia es un elemento muy importante para completar el riesgo de transmisión vectorial de la enfermedad de Chagas en esta región, y por lo tanto de especial interés para el departamento del Putumayo, debido a que se han realizado pocos estudios y que se cuenta con las condiciones ecológicas óptimas para los vectores y reservorios de esta enfermedad. Cabe resaltar la importancia que tiene las acciones de Vigilancia entomológica de la Secretaría de Salud del Putumayo, porque permiten aumentar el registro de nuevas especies de triatominos del país y aumentar de manera considerable el número de registros en nuevas localidades, como también el uso de mecanismos para combatir los vectores, romper el ciclo y así evitar la transmisión del parásito.

\section{Agradecimientos}

A la Dra. Sorrel Parisa Aroca Rodríguez, gobernadora del departamento del Putumayo 2016 - 2019; a la Dra. Zulma Carina Hernández, secretaria de salud departamental del año 2019; a la Dra. Ana Cristina Hidalgo P. E Grupo salud Pública, a la Dra. Ana Carolina Villota P. U Laboratorio de Salud Pública - febrero de 2019, por las gestiones y apoyo en la 
Primer registro de Eratyrus mucronatus Stal, 1859: vector de la enfermedad de

Chagas en el departamento del Putumayo, Colombia

realización de las acciones de Vigilancia Entomológica en el departamento del Putumayo; a los auxiliares del Programa de Enfermedades Transmitidas por Vectores por el apoyo en el trabajo de campo y al Grupo de Entomología de la Subdirección Red Nacional de Laboratorios del Instituto Nacional de Salud por su apoyo en la confirmación de los especímenes.

\section{Referencias}

1. La enfermedad de Chagas (tripanosomiasis americana) [Internet]. Organización Mundial de la Salud. 2018 [citado 13 de septiembre de 2018]. Recuperado a partir de: http://www.who.int/es/news-room/ fact-sheets/detail/chagas-disease-(american-trypanosomiasis)

2. World Health Organization. Research priorities for Chagas disease, human African trypanosomiasis and leihsmaniasis [Internet]. World Health Organ Tech Rep Ser. [citado 13 de septiembre de 2018] 2012; 975: 1-100. Recuperado a partir de: https://apps. who.int/iris/bitstream/handle/10665/77472/WHO TRS 975 eng.pdf;jsessionid=92E38E737549D9D3E2D9A64A9B809E0D?sequence $=1$

3. SIVIGILA, Sistema de Vigilancia Epidemiológica 2012 a semana 45 de 2018 [Internet]. Colombia, SE 45 de 2018 [citado 13 de septiembre de 2018]. Recuperado a partir de: https:/www.ins.gov.co/buscador-eventos/BoletinEpidemiologico/2018\%20 Bolet $\%$ C3\%ADn $\% 20$ epidemiol $\%$ C3\%B3gico $\% 20$ semana \%2045.pdf

4. La enfermedad de Chagas (tripanosomiasis americana) [Internet]. Organización Mundial de la Salud OMS; 2018 [citado 13 de septiembre de 2018]. Recuperadoa partir de https://www.who.int/es/news$\mathrm{room} /$ fact-sheets/detail/chagas-disease-(american-rypanosomiasis).

5. Magallon et al. Distribución de los vectores de la enfermedad de chagas (Hemiptera: Reduviidae: Triatominae), en el estado de Jalisco, Mexico. Rev. Biomed. 1998. 9:151-157.

6. Plan de Desarrollo "Putumayo Territorio de Paz, Biodiverso, y Ancestral. Juntos podemos Transformar" 2016-2019 p. 22

7. Estrategia de Gestión Integrada para la promoción, prevención y control de las Enfermedades Transmitidas por Vectores en el Departamento del Putumayo [Internet]. EGI - ETV Secretaría De Salud 2012 2021 [citado 13 de septiembre de 2018]. Recuperado a partir de: http://www.siellano.com/secretariasaludpalmira/images/pdf/informes/DocumentoEGI-ETVPalmira2016.pdf
8. Volver a creer para crecer [Internet]. Plan de desarrollo del municipio de Orito-Putumayo para el periodo 2012 - 2015 [citado 13 de septiembre de 2018]. Recuperado a partir de: https://www.putumayo.gov. co/images/documentos/PDMunicipales/PDM Orito2012 2015.pdf

9. Schofield C. Triatominae: Biología y Control. West Sussex. United Kingdom: Eurocommunica Publications. 1994.

10. Carcavallo RU, Galíndez I, Jurberg J, Galvao and Lent H. Pictorial keys for tribes, genera and species of the subfamily Triatominae. In Carcavallo RU, Galíndez I, Jurberg J, Lent H (eds). Atlas of Chagas' Disease Vectors in the Americas, Vol I. Rio de Janeiro: Editora Fiocruz. 1997. p 107-244.

11. Guhl F; Aguilera, G; Pinto N; Vergara D. Actualización de la distribución geográfica y ecoepidemiológica de la fauna de triatominos (Reduviidae: Triatominae) en Colombia. Biomédica. 2007;27(Supl. 1): 143-62. doi: 10.7705/biomedica.v27i1.258.

12. Barreto M, Burbano M.E, Barreto P. Nuevos datos sobre la distribución de Triatominae (Hemiptera: Reduviidae) en el departamento de Putumayo, Colombia. Memorias XI Congreso Colombiano de Parasitología y Medicina Tropical. Biomédica. 2003; 23:1:101.

13. Vásquez L, Jaramillo E, Molina J. Triatominos del Municipio de Mocoa departamento del Putumayo. Revista de la Facultad de Ciencias de la Salud de la Universidad del Cauca [Internet] 2007 [citado 13 de septiembre de 2018]; 46-50. Recuperado a partir de: http://revistas.unicauca.edu.co/index.php/rfcs/ article/view/884

14. Guhl F, Marinkelle CJ, Becerra W, Romero C. Nuevos registros de triatominos en Colombia. Biomédica. 1991; 11:90-1.

15. Castillo, D y Wolff, M. Aspecto del comportamiento de los triatominos (Hemiptera: reduviidae), vectores de la enfermedad de chagas. Biomédica. 2000; 20:59-64. P61.

16. Sherlock I, Carcaballo R, Galíndez I. List of natural and experimental flagellates infections in several Triatominae species. In: Carcavallo RU, Galíndez I, Jurberg J, Lent H (eds), Atlas of Chagas' Disease Vectors in the Americas, Vol I. Rio de Janeiro: Editora Fiocruz; 1997. p. 289-298.

17. Guerrero L, Scorza JV. 1981. Las fuentes alimenticias de algunos Triatominae silvestres en los llanos centro occidentales de Venezuela. Bol Dir Malariol y San Amb 21(2):129-139. 
18. Perruolo G, Morales O. 1987. Reservorios de la enfermedad de Chagas en el estado Táchira, Venezuela (zona norte). Bol Dir Malariol y San Amb. 28:(1 y 4) 1-7.

19. Noireau F, Bosseno M, Carrasco R, Talleria J, Vargas F, Camacho C, et al. Sylvatic triatomines (Hemiptera: Reduviidae) and possible infection with Trypanosoma cruzi (Kinetoplastida: Trypanosomatidae). J Med Entomol. 1995; 32:(5) 594 - 598.

20. Caravallo RU, Rodriguez M, Salvatella R, Curto S, Scherlock I, Galvao C. et al. Habitats and related fauna. In Carcavallo RU, Galíndez I, Jurberg J, Lent $\mathrm{H}$ (eds), Atlas of Chagas' Disease Vectors in the Americas, Vol II. Rio de Janeiro: Editora Fiocruz. 1998 p 561-619.
21. Molina J, Gualdron L, Brochero H, Olano V, Barrios D, Guhl F. Distribución actual e importancia epidemiológica de las especies de triatominos (Reduviidae, Triatominae) en Colombia. Biomédica. 2000; 20:344-360.

22. Dias P, Diotaiuti L. Vectores secundarios de la enfermedad de Chagas en el Brasil y perspectivas para su control. En Guhl F, Jaramillo C, (eds), Curso Taller Control de Tripanosomosis Americana y Leishmaniosis: Aspectos Biológicos, Genéticos y Moleculares. Bogotá: Editorial LTDA; 1998. p 154-159. 